Case Report

\title{
Inguinoscrotal bladder hernia: incidental finding on bone scintigraphy
}

\begin{abstract}
Inguinoscrotal hernia of the bladder is relatively uncommon, difficult to diagnose and remain a surgical challenge. ${ }^{1,2,3,4}$ We report a 72-years-old man with a recent diagnosis of prostate neoplasm who underwent a Technetium-99 hidroximethylene-diphosphonate (Tc99m HMDP) bone scintigraphy which reveals, as an incidental finding, a left inguinoscrotal hernia containing a portion of the urinary bladder.
\end{abstract}

Keywords: bone scintigraphy, inguinal bladder hernia, Technetium-99m hidroximethylene-diphosphonate, inguinoscrotal hernia, urinary bladder
Volume 7 Issue 3 - 2020

\author{
Anderson Cardozo-Saavedra,' Diego \\ Villasboas-Rosciolesi,' Eliana Carrillo- \\ Villamizar,' Sandra Menéndez-Sánchez,' \\ Ruben Bellviure-Meiro,' Amparo García- \\ Burillo,' Joan Castell-Conesa ${ }^{2}$ \\ 'Department of Nuclear Medicine,Vall d'Hebron University \\ Hospital, Barcelona, Spain \\ ${ }^{2}$ Faculty of Medicine, Universitat Autònoma de Barcelona, \\ Barcelona, Spain
}

Correspondence: Anderson Cardozo-Saavedra, Department of Nuclear Medicine, Vall d'Hebron University Hospital, Barcelona, Spain, Email andersoncardozmd@gmail.com

Received: June 04, 2020 | Published: June 17, 2020

\section{Introduction}

Imaging techniques, including nuclear medicine examinations, often give rise to findings that are incidental and unrelated to the reason the study was prescribed. It's necessary the right diagnostic handling of these findings, in order both to avoid unnecessary procedures and to identify potentially risk conditions. Inguinoscrotal hernia of the bladder is relatively uncommon (less than $4 \%$ of inguinal hernias). ${ }^{1-3}$ It usually presents without specific symptoms and it is discovered in less than $10 \%$ of cases prior to an operation. As they are often difficult to diagnose and remain a surgical challenge, preoperative imaging is essential to prevent complications. ${ }^{4}$

\section{Case}

A 72-years-old man with obesity and a recent diagnosis of prostate cancer, was referred to our department for staging, and a $99 \mathrm{mTc}-$
HMDP bone scintigraphy was performed. The whole-body images (Figure 1A) revealed no evidence of bone metastasis, but an abnormal accumulation of the radiopharmaceutical was observed in the left anterior extra-pelvic region (black arrows). The lateral pelvic view (Figure 1B) localized the focus on the inguinoscrotal area. It was identified as an inguinoscrotal hernia of the bladder (IHB) by a pelvic MRI. Selected T2W-MRI sagital and axial slices (Figure 1C\&Figure 2) show the urinary bladder (white arrows) and the IHB (black arrow) images in a concordant disposition to the radioactive foci seen in the bone scan (Figure 1B). On physical examination, presented a bilateral inguinal bulge with predominance of the left side, which was more noticeable after weight loss. This bulge worsened with the Valsalva maneuvers and with the bladder filling, and decreased after each urination.

Our patient has been clinically followed without surgical treatment to date.

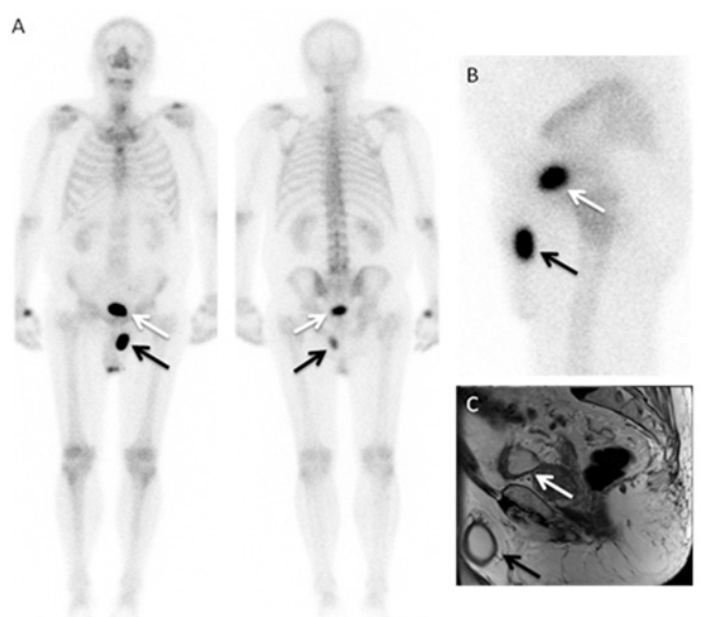

Figure I A. Bone scan planar, anterior and posterior, whole body images after intravenous injection of $925 \mathrm{Mbq}$ of $99 \mathrm{mTc}-\mathrm{HMDP}$, performed according to the standard protocol, show an accumulation of the radiotracer in the left anterior extra-pelvic region (black arrows), with a similar intensity to that of the bladder (white arrows), B. Bone scan lateral pelvic view. C. A selected T2W-MRI sagital slice, shows similar findings. 


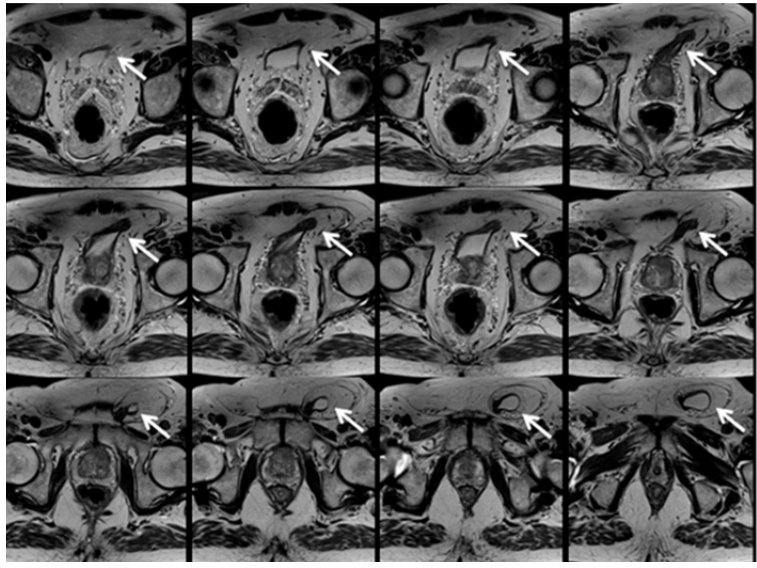

Figure 2 Some T2W-Pelvic MRI transversal slices showing the inclusion of the supero-lateral region of the urinary bladder (white arrows) in the left inguinal hernia.

\section{Discussion}

IHB occurs in an acquired direct inguinal hernia with the bladder pulled into the hernia, together with a sheath of peritoneum, which forms its sac. This kind of hernia represent $0.5-3 \%$ of lower abdominal hernias. They mostly occur in men older than 50 years, and associated risk factors include overweight, chronic urinary obstruction, and a weak pelvic musculature. ${ }^{4}$ Symptoms are heterogeneous and generally include inguinal swelling associated or not with pain or voiding urinary tract symptoms. ${ }^{1}$ The majority of patients are asymptomatic and are usually diagnosed incidentally in imaging studies or during inguinal hernia surgery. ${ }^{5}$ Radiographic imaging is not routinely performed in the workup of inguinal hernias, but cystography is the gold standard in diagnosis with the highest diagnostic value showing indentation of the bladder wall. ${ }^{6}$ There have been a few case reports about nuclear medicine diagnosis of this condition and, for clinicians and nuclear medicine specialists, its important being aware about this finding as a possible pitfall in planar bone scan image interpretation and as relevant additional information about the patient. ${ }^{8-11}$

\section{Conclusion}

This kind of cases show up the relevance of additional views and anatomic imaging comparison to interpret atypical findings in planar bone scan images, besides making an accurate diagnosis and avoiding surgical complications. ${ }^{9}$

\section{Acknowledgments}

None.

\section{Funding}

Self funding.

\section{Conflicts of interest}

Author declares that there is no conflict of interest.

\section{References}

1. Branchu B, Renard Y, Larre S, et al. Diagnosis and treatment of inguinal hernia of the bladder: a systematic review of the past 10 years. Turk J Urol 2018;44(5):384-388.

2. Koontz AR. Sliding hernia of diverticula of bladder. Arch Surg. $1955 ; 70: 436-438$

3. Madden JL, Hakim S, and Agorogiannis AB: The anatomy and repair of inguinal hernias. Surg Clin North Am. 1971;51:1269-1292.

4. Ayaaz Habib. A Rare Case of Inguinal Hernia with Complete Bladder Herniation. Case Rep Surg. 2017;2017:4658169.

5. Oruç MT, Akbulut Z, Ozozan O, et al. Urological findings in inguinal hernias: a case report and review of the literature. Hernia J Hernias Abdom Wall Surg. 2004;8:76-79.

6. Moufid K, Touiti D, Mohamed L. Inguinal bladder hernia: four case analyses. Rev Urol. 2013;15(1):32-36.

7. Sarr A, Ondo CZ, Sow Y, et al. Inguinal hernia of the bladder: about 8 cases. Pan Afr Med J. 2015;22:7.

8. Ji T, Gao D, Chen B, et al. 99mTc-MDP uptake in SPECT/CT by a bladder hernia simulating inguinal metastasis: A case report. Oncology letters. 2016;11(2):1398-1400

9. Goel S, Gadiraju R. Left inguinal hernia mimicking metastasis on bone scan. Radiol Case Rep. 2015;8(4):559.

10. Iagaru A, Siegel M. Demonstration of a right inguinal hernia containing urinary bladder diverticulum on whole-body bone scan and pelvic CT. Eur J Nucl Med Mol Imaging. 2006;33(2):234.

11. De Vincentis G, Pontico M, Ricci M, et al. Incidental findings in traditional nuclear medicine practice. J Radiol Oncol. 2018;2:036-040. 